EPJ Web of Conferences 70, 00039 (2014)

DOI: 10.1051/epjconf/20147000039

(C) Owned by the authors, published by EDP Sciences, 2014

\title{
Transaction and Non Locality in Quantum Field Theory
}

\author{
Ignazio Licata ${ }^{1, a}$ \\ ${ }^{1}$ Institute for Scientific Methodology, Palermo, Italy
}

\begin{abstract}
The most part of the debates on Quantum Mechanics (QM) interpretation come out from the remains of a classical language based upon waves and particles. Such problems can find a decisive clarification in Quantum Field Theory (QFT), where the concept of "classical object" is replaced by an interaction networks. On the other hand, it is simpler to discuss about non-locality in QM than in QFT. We propose here the concept of transaction as a connection between the QM and QFT language as well as the possibility to introduce quantum non-locality ab initio. We also mention the cosmological consequence of a non-local archaic vacuum here defined
\end{abstract}

\section{Introduction}

Why are people, magazines (and philosophers too) still back to 1927 and talk about waves and particles, while the theoretical physicists talk about quantum field theory?

Since its appearing Quantum Theory has put radical questions which have challenged not only Classical Physics, but the structure itself of the "explanation" concept born within the "continuous", "dynamical" and "evolutionary" western thinking tradition. Let's remember what Rutherford asked to Bohr: "How does an electron know which orbit to jump in?" And later Schrödinger "If these bloody quantum jumps exist, I'll regret having studied Quantum Mechanics!'”. Non-locality problem was already well put in both statements. Copenhagen Interpretation has hidden - so to say - non-locality behind the statistical machinery of "casuality", but after the experimental works by V. A. Rapisarda, A. Aspect and A. Zeilinger (just to name some), we have to admit that non-locality is at the core of Quantum Physics and it should be introduced ab initio within the theory structure not as a later strangeness, but as a fundamental principle. In the same way as the Einstein's Covariance Principle puts the equivalence between inertial and gravitational mass at the GR foundation. Understanding the centrality of non-locality could solve the old debate on "Quantum Mechanics foundations". A debate which too often seems to be stopped back to 1927 (nineteen twenty seven), whereas it should be rather include the most beautiful and ripe fruit of Quantum Mechanics: the Quantum Field Theory, and Quantum Cosmology too.

The first one because it is necessary to speak about interactions, considering they are the only object of our observations, the second one because we need a theory able to tell how quantum vacuum is "switched on" and gives birth to some objects and dynamics and not some other ones. That Informational matrix Wheeler called It from (Q) Bit [18]

\footnotetext{
ae-mail: ignazio.licata@ejtp.info
} 
Since the non-local correlations do not transport energy, they do not violate Relativity. This situation goes under the name of "peaceful coexistence" between Relativity and Quantum Physics and has suggested that the unification between QM and GR can happen within a geometro-dynamic program inspired to the GR philosophy, where the QM could be incorporated as a conformal "deformation" of space-time.The geometric approaches to quantum processes are based on Quantum Potential and Weyl's geometry, using a modification of the Weyl-Dirac theory [19, 16, 14, 13]

In spite of some interesting results, actually, once again, things are not so simple. Non-locality remains a phenomenon that rests uncomfortably on a "mechanical" vision of the universe. As Heisenberg observed at the dawning of quantum theory, the quantum events are radically a-causal, and cannot therefore be retraced into the traditional Einsteinian space-time arena, however "extended" it may be.

\section{The "particle" concept from a Quantum Field Theory Point of View}

The Quantum Field Theory is the mature daughter of QM and the most general syntax we know to describe forces. It replaces the "hard" naïve particle of classical physics and QM with a network of interactions. It greatly modifies the traditional vision of "permanent object". Let's remember some essential points:

a) The physical world is described as a discrete net of interaction vertices where some properties (space-time position, quadri-impulse, spin, etc.) are destroyed and created. Such properties' measurement is all that we know of the physical world from an operational view point. Any other construction in Physics - like the continuous space-time notion itself or the evolution operators - has the role to causally connect the measured properties. So we can say they are" emergent" with respect to the network of events;

b) Heisenberg uncertainty principle in its more general and "right" QFT form-, phase for number of quanta, $\Delta n \Delta \phi \geq h$ - does not indicate the limits of measurement between classical variables, but the applicability limit of the continuous space-time concept. In phenomena involving a few number of potential "impacts" (interaction vertices) the representation of the field as a continuous propagation in a space-time environment is no more applicable [see 15, 12];

c) Motion is no more a continuous phenomenon, but a discontinuous process in the spacetime coordinates. The are no more "objects" as exclusive bearers of permanent "qualities". QFT introduces - by the discontinuity and the relativistic invariance - non-locality as a basic ingredient. The propagation of physical quantities in the space-time appears more similar to the phenomenon of switching on a line of blinking bulbs, or dislocation in a crystal.

If, in a quantum leap, the quantum state " $i$ " is destroyed and the new quantum state " $\mathrm{j}$ " is created the element $X(i, j)$ of the physical quantity $X$ is involved. Only when the matrix $X(i, j)$ is diagonal the interaction will leave the $X$ value unchanged and that leap can then be considered as a measurement of $\mathrm{X}$. So the quantum laws have generally a matrix-like form and it also follows the existence of quantities not simultaneously defined. This frame modifies quite widely the semi-classical vision associated to QM.

On the other hand, let's remember that the "heuristic" vision of the quantum as a "particle" was introduced by Einstein (photoelectric effect) and later reprised by Compton (electron/photon scattering), but it has no citizenship in the original Planck formulation, where it is already more similar to a transaction, as well as, indeed, in the quantum jump of the later atomic theory. As a matter of fact, quantum jump has been the first genuine non-locality manifestation in Physics.

According to such view there is no strangeness in the Afshar Experiment [1]

Afshar objective was to confute the complementarity principle, a Bohr philosophical invention useful in the early QM formulation, by modifying the double slit experiment and individuating a situation 
where the photon propagation shows both corpuscular and ondulatory features. Anyway Afshar insisted in the possibility to individuate what slit the photon had passed and everybody was concerned in demolishing such statement [see for ex. 13, 17].

From the QFT viewpoint it is easy to consider Afshar experiment as a ondulatory-corpuscular mixed situation typical of the field modes, and in this sense, as J. Cramer stated, it can actually be considered "a farewell to Copenhagen".

\section{What "interpretation" of QM in the Light of QFT ?}

The most part of Quantum Physics' Interpretations persists in deriving non-locality from local situations by using continuous concepts such as space-time or the environment, so running the risk to sail along the ether ghost or paradoxes flavouring of Alice in Wonderland.

The ondulatory language and statistical interpretation can work only when a great number of (even virtual) interaction vertices is involved. For example, the ground state of an hydrogen atom is really a sort of average on many virtual interactions between the nuclear electric field and the orbital electron; this undergoes to many virtual destructions/creations and QM "electron" is really the conserved net result of these processes. In this sense QM is a good approximation of QFT for systems at low energy when the number of quanta is conserved. In QFT the field of a single electron becomes the field of the whole Universe electrons, and its second-quantized wave function can admit different numbers of electrons and can have a superposition between states with different number of particles. This confirms that particles are not "persistent objects" but networks of transactions.

Now let's try to understand what kind of reading of the QM is fixed by QFT. Following Penrose terminology, the QM structure is given by $\mathrm{U}$ (evolution operators) and R (“clicks" or single events), and its so-called foundational problems come out from the difficulty to connect $U$ and R. In particular, R "collapse" has often been regarded as the Euclid's "fifth postulate" for the impossibility to derive it from U. An alternative way is to conciliate complementary $\mathrm{U}$ and R by means of the famous "pilot wave", which leads to the well-known problems with the "meaning" of $\Psi$. We know, from QFT, that the physical world is a net of energetic transitions and our speaking of waves/particles is only an approximated language .

The reading of QM which best fits this approach is the new transactional theory [5]. Its first version, owed to Cramer [6], regarded the non-local connection as a handshaking between anticipated and delayed potentials a la Wheeler-Feynman, which thing arouse a lot of mathematical and conceptual problems connected to the still too classical context. Intuitively the idea was quite simple: each particle "responded" to all of its future possibilities. It is obviously a space-time language. In the new version no complication of this kind comes out, just some simple rules about the transactions' opening and closing, so to fix in univocal way the evolution operators. Actually, at a fundamental level only the transactions between field modes take place, and the wave-function simply reveals as a statistical coverage of a great amount of elementary transitions.

We can imagine the Vacuum not only as an eigenstate of minimum energy, but also the network of all the possible transactions of the field modes in an "undivided Oneness", and it has to be regarded as a radical non-local and event-symmetric state [2]. Planck constant is then the measure of the fabric "elasticity". The vacuum constrains and conveys the dynamical processes we observe. It is a fabric from which patterns emerge by $\mathrm{R}$ processes and such patterns influence the vacuum activity, in a quantum feedback. On this basis we can think that in the future the Bell's Theorem not only will tell us the limits of hidden variables theories, but will be the cornerstone of a theory able to explain nonlocality as a residual effect, in particular conditions, from the manifestations of the primeval vacuum. The "laboratory" non-locality appears as a particular case of this atemporal wholeness. 


\section{A short glance inside transactions, or: how to introduce non-locality in QFT}

QFT language is centered on elementary terms which are the creation and destruction operators.

Starting from such terms we build the commutation and anti-commutation relations which determine the single field features (for ex. fermionic or bosonic ones). Then the Hamiltonian describing the evolution of the free fields and the interacting ones are introduced. The transactional proposal does not touch such scheme, but fixes the general condition to introduce non-locality at the heart of QFT. Speaking in a more general way, we shall have at $t=t_{1}$ the event of the creation-destruction of a quality $Q(|\mathrm{Q}><\mathrm{Q}|)$ and at $t=t_{2}$ the event of the creation-destruction of a quality $R(|\mathrm{R}><\mathrm{R}|)$ These two processes will be linked by a time evolution operator $S$ according to the ring:

$$
\begin{aligned}
& |Q>\quad<Q| \quad t=t_{1} \\
& S \downarrow \quad \uparrow \quad S^{+} \\
& |R>\quad<R| \quad t=t_{2}
\end{aligned}
$$

In other words, | Q $>$ is transported from $S$ into $\mid Q^{\prime}>$ and projected into $<\mathrm{R}|,| \mathrm{R}>$ is transported by $S^{+}$into $\mid R^{\prime}>$ and projected onto $<\mathrm{Q} \mid$. The amplitudes product :

$$
<R|S| Q><Q\left|S^{+}\right| R>=|<R| S|Q>|^{2}
$$

is immediately obtained, which is the probability of the entire process. If quality $Q$ is constituted by a complete set of constants of motion then $R=Q$ and this is the type of process which can describe the propagation of a photon-type quantum, otherwise it is the generic process of the creation of a quality $Q$ causally linked (by means of $S$ ) to the destruction of a quality $R$. Moving to the representation of the coordinates, by substituting bras and kets with wavefunctions, we once again obtain as a particular case the result already seen with the well know Schröedinger non-relativistic expressions.

From an algebraic point of view, the transactional ring is a sort of identity operator, because $S S^{+}=$ $S^{+} S=1$ and the qualities $Q, R$ are simultaneously created and destroyed. This is the by now classic case of EPR and GHZ phenomena. One has the impression that every quantum process (therefore all matter) and time itself are emitted from an invariant substratum and re-absorbed within it. We propose to call such substratum "archaic vacuum" to distinguish it from the QFT traditional dynamic vacuum and to indicate all the self-consistency logical constraints which rule the "fabric of reality".

As an example of a transactional network, let us consider a well known process in QFT, constituted by the decay of a microsystem, prepared in the initial state 1, into two microsystems 2,3 which are subsequently detected. The preparation consists of destroying the quality 1 [which we shall indicate by $(1 \mid$ ] which closes the transaction which precedes it, and creating the quality 1 [which we shall indicate by |1)] which opens a new transaction. It will be represented by the form |1)(1|.

The decay consists of destroying the qualities 2, 3 which close the transaction started at the preparation state, and creating the qualities 2, 3 which open a new transaction which will be closed at the detection of microsystems 2 , 3. We represent it by the form [| 2)|3)][(2|(3)].

The detection of microsystems 2, 3 will be made by the destructions of qualities 2, 3 which close the transaction started at the decay, and by the creations of qualities 2,3 which open subsequent transactions. It will be represented by the interaction events $\mid 2)(2|| 3),(3 \mid$.

The double transaction here described corresponds to the process usually associated to the probability amplitude $<2,3|\mathrm{~S}| 1>$.

Another example is Young's classical double slit experiment. The preparation of the particle initial state can be represented by the form $\mid 1)(1 \mid$, following the same reasoning as above. Instead of the 
decay, here we have the crossing of slits 2 and 3, i.e. the interaction between a particle and a double slit screen represented by [| 2$) \mid 3)][(2 \mid(3 \mid]$. Instead of the detection of the two particles created in the decay, here we only have the detection of the particle on the second screen at a certain position 4, i.e. : |4)(4|. Two transactions are involved: the first starts with the preparation of the particle and ends with its interaction with the first screen; the second begins with this second interaction and ends with the interaction of the particle with the second screen. The second interaction then constitutes the beginning of the following transaction. The process is that which corresponds to the probability amplitude $<4|\mathrm{~S}| 1>$.

We note that the forward time evolution of the amplitudes, represented by $\mathrm{S} \mid 1>$, contains both the kets $|2>| 3>$,; nevertheless, processes relating to the passage through the individual slit $a$ (where $a=2,3)$ do not exist. Such processes would require an intermediate event represented by $\mid a)(a \mid$, which actually does not take place. It is in this sense that the processes which could be associated to compound probability amplitudes $<4|\mathrm{~S}| \mathrm{a}><\mathrm{a}|\mathrm{S}| 1>$ are "virtual" and not real. The process of the crossing of one of the two slits becomes real when the other slit is closed.

\section{Quantum Pre-Space in de Sitter Cosmology}

The description of the relation between vacuum and its manifestations is provided by Bohm theory of holomovement between explicate and implicate order [3]. It is the passage, which can be formalized by Green operator and Wick Rotation, from the timeless non-local to the time and local in a continuous feedback. It is important to understand that holomovement does not happen "in the time" but gives origin to the space-time itself by the activity of the transactions. From the definition of holomovement in terms of Green function it is easy to derive the Heisenberg's form of the Schrödinger Equation [7].

The irreducible nature of a single event - irreducible for the statistical interpretation - justifies the introduction of casuality. It is the quantum active information inaccessible in the explicate order which prevents us from following the single event [9]. Thanks to non-locality, the quantum casuality becomes intelligible, but not less radical. Einstein was right, and Bohr too!!!

There are many images and famous metaphors, more or less mathematical, to indicate the relation between implicate and explicate order, such as the hologram one. Here we have chosen a Mandelbrot fractal to remember the difference between dynamical causality in the explicate order and formal causality in the implicate order. The non-local features are linked to the second one, as the initial condition in cosmology.

Cosmology is not a separate branch of Physics, but the global history of matter. It is the logic activation of vacuum in the primeval informational matrix and the formal causality that "switches on" and guides the evolution of the physical world. On the other hand, we cannot avoid asking what the vacuum global form is and what kind of global/local relations it rules.

The old Big Bang conception as a "thermodynamic balloon" seems to be irreparably compromised by now. We just quote here the Author and L. Chiatti work on Archaic Universe where the starting point is the group approach to the DeSitter Universe as quantum vacuum's geometrical shape [10,11]. The elimination of the initial singularity by the adoption of de Sitter 5 hyper-sphere as pre-space makes possible a very concise description of the boundary conditions necessary for the evolution of the observed physical universe. Such "pre-space" we define as "archaic" has not to be considered as antecedent to "Big-Bang", but rather as a pre-spatial and a-temporal substrate of the usual spacetime metric containing in nuce all the evolutionary possibilities that the General Projective Relativity (GPR) equations indicate. After eliminating any geometrical singularity with Euclidean substrate, the description of the Universe evolution can be seen as an extended nucleation from a coherent timeless 
state (de Sitter isotropic singularity) with very high non-local information to an observable mix of local matter-energy. The passage from the archaic to the evolutionary state is defined by a sort of "holomovement" due to a Wick rotation which characterizes the appearance of the dynamics and time arrow starting from the general constraints on the pre-dynamic, archaic condition. It is remarkable that the theory acts as an "Occam razor" on many speculations about dark matter and inflation, gives a purely geometrical description to the cosmological constant and introduces, by the cosmological constant, new relations between macro and micro-physics as a new kind of cosmological non-locality. In particolar, the study of transactions in Projective General Relativity has shown the existence of a minimum time of transaction - similar to Caldirola chronon - of $\theta_{0} \approx 10^{-23} \mathrm{sec}$..

If this duration exists, all physical phenomena of shorter duration than it must link events which are not extremes of transactions and which therefore are not $\mathrm{R}$ processes. These phenomena must therefore, according to Penrose's terminology, constitute aspects of a U process.

In terms of quantum physics everyday language, the linked events are therefore extreme vertices of "virtual" propagations that exist solely as terms of the expansion of the time evolution operator of the system being studied. In the cosmological approach under consideration, quantum non-locality leads to cosmological non-locality as a global constrain on the distribution of the creation/annihilation events on the 5-sphere, which is to say on the physical Universe's possible histories. If we would describe the origin by the language of dynamics, we could say that the thermodynamic equilibrium is reached in a time equal to the 5-sphere radius divided by $c$, and then inflated to cosmic dimensions, a description very similar to the inflationary mechanism [4].

So to overcome some difficulties in microphysics and cosmology it seems to be necessary to introduce - together with the traditional dynamic vacuum - an archaic vacuum as the expression of a generative order of physical reality.

\section{References}

[1] S. S. Afshar, E. Flores, K. F. McDonald, E.Knoesel, Found. of Physics 37, 2007 295-305

[2] D. Bohm, Wholeness and the Implicate Order, (Routledge, London 1980)

[3] D. Bohm, B. Hiley, The Undivided Universe: An ontological interpretation of quantum theory, (Routledge, London 1993)

[4] L. Chiatti, in Vision of Oneness, I. Licata and A. Sakaji eds, (Aracne Publ., 2011)

[5] L. Chiatti, arXiv: 1204.6636 (2012)

[6] J. G. Cramer, Int. Jour. of Theor. Phys. 27, 1988 227-236

[7] B. Hiley, in New Structures for Physics, B. Coecke ed (Springer, 2011)

[8] R. Kastner, Studies in History and Philosophy of Modern Physics 36(4), 2005 649-658

[9] I. Licata, in Physics of Emergence and Organization, I. Licata and A.Sakaji eds, (World Scientific 2008)

[10] I. Licata, L. Chiatti, Int. Jour. of Theor. Phys., 48(4), 2009 1003-1018

[11] I. Licata, L. Chiatti, Int. Jour. of Theor. Phys., 49(10), 2010 2379-2402

[12] I. Licata, in Vision of Oneness, I. Licata and A. Sakaji eds, (Aracne Publ., Rome 2011)

[13] I. Licata, D. Fiscaletti, Int. Jour. of Theor. Phys., Online, DOI: 10.1007/s10773-012-1245-0 2012

[14] M. Novello, J. M. Salim, F. T. Falciano, IJGMMP 8(1), 2011 87-98

[15] G. Preparata, in An Introduction to a Realistic Quantum Physics, (World Scientific, 2002)

[16] A. Shojai, F. Shojai, Classical and Quantum Gravity,, 21(1), 2004 1-9

[17] O. Steuernagel, Found. of Phys., 37(9), 20071370 


\section{ICFP 2012}

[18] J. A. Wheeler, in Complexity, Entropy, and the Physics of Information, W. Zurek ed,(AddisonWesley, Redwood City 1990)

[19] W. R Wood, G. Papini, in The Present Status of Quantum Theory of Light, S. Jeffers, S. Roy, J.

P. Vigier , G. Hunter eds, (Springer 1996) 